Pesq. Vet. Bras. 37(8):897-903, agosto 2017

DOI: $10.1590 / \mathrm{S} 0100-736 \mathrm{X} 2017000800020$

\title{
Morfologia da traqueia e lobação pulmonar de Leopardus pardalis (jaguatirica) ${ }^{1}$
}

\author{
Cristiano de O. Schuingues ${ }^{2 *}$, Mendelson G. de Lima ${ }^{3}$, Geise H.R. Sardinha ${ }^{5}$, Adriano \\ L. Pimenta ${ }^{5}$, Cléia C. Monteiro ${ }^{5}$, Carlos E. Ambrósio ${ }^{4}$, Daniele dos S. Martins ${ }^{4}$ \\ e Gerlane de M. Costa ${ }^{3}$
}

\begin{abstract}
Schuingues C.O., Lima M.G., Sardinha G.H.R., Pimenta A.L., Monteiro C. C., Ambrósio C.E., Martins D.S. \& Costa G.M. 2017. [Morphology of the trachea and lung lobation of Leopardus pardalis (ocelot).] Morfologia da traqueia e lobação pulmonar de Leopardus pardalis (jaguatirica). Pesquisa Veterinária Brasileira 37(8):897-903. Programa de Biodiversidade e Agroecossistemas Amazônicos, Universidade do Estado do Mato Grosso, Campus I, Rodovia MT-208 Km 146, Alta Floresta, MT 78580-000, Brazil. E-mail: cristiano-af12@hotmail.com

The species Leopardus pardalis, popularly known as ocelot, is a medium sized cat with wide distribution in the national territory and is included in the list of species of Brazilian fauna threatened with extinction. This study aimed to characterize the macroscopic and microscopic aspects of the trachea and lung lobes in L. pardalis. Analyses were performed using five copies of ocelot, two males and three females, adults, three of these specimens died from being run over on highways nearby the municipality of Alta Floresta, and two donated after death by IBAMA to the Laboratory of Zoology and Animal Morphology. In the trachea specimens was a flexible tube formed by pairs of cartilaginous arches, with pseudo-stratified epithelium ciliated not in its inner lining, and other basic structures of this body. Lung proved as a body made up of lobes separated by cracks with a usual parenchyma to the other described mammals. With respect to the anatomic features of the trachea and lung were similar to other mammals, as in the microscopic aspects of the trachea showed certain peculiarities found differing from the other jobs. Regarding the appearance of the lung parenchyma histological characteristics did not differ with those described.
\end{abstract}

INDEX TERMS: Trachea, lung, Leopardus pardalis, ocelot, bronchial tree, felines, pulmonary parenchyma, respiratory system.

RESUMO.- A espécie Leopardus pardalis, conhecida popularmente como jaguatirica, é um felino de médio porte com ampla distribuição em território nacional e está incluída na lista de espécies da fauna brasileira ameaçadas de extinção. Este estudo teve como objetivo caracterizar os aspec-

\footnotetext{
${ }^{1}$ Recebido em 26 de agosto de 2016.

Aceito para publicação em 11 de janeiro de 2017.

${ }^{2}$ Mestrando, Programa de Biodiversidade e Agroecossistemas Amazônicos, Universidade do Estado de Mato Grosso (UEMT), Campus I, Rodovia MT-208 Km 146, Alta Floresta, MT 78580-000, Brasil. *Autor para correspondência: cristiano-af12@hotmail.com

${ }^{3}$ Faculdade de Ciências Biológicas e Agrárias, UEMT, Campus I, Rodovia MT-208 Km 146, Alta Floresta, MT 78580-000, Brasil.

${ }^{4}$ Departamento de Medicina Veterinária, Faculdade de Zootecnia e Engenharia de Alimentos (FZEA), Campus da Universidade de São Paulo (USP), Av. Duque de Caxias Norte 225, Pirassununga, SP 13635-900, Brasil.

${ }^{5}$ Acadêmicos do Curso de Licenciatura e Bacharelado em Ciências Biológicas, UEMT, Campus I, Rodovia MT- 208 Km 146, Alta Floresta, MT 78580-000, Brasil.
}

tos macroscópicos e microscópico da traqueia e lobos pulmonares em L. pardalis. As análises foram feitas utilizando cinco exemplares de jaguatirica, sendo dois machos e três fêmeas, adultos, três desses exemplares foram a óbito por atropelamento em rodovias próximas ao município de Alta Floresta, e dois doados após a morte pelo IBAMA ao o Laboratório de Zoologia e Morfologia Animal. Nos espécimes a traqueia era um tubo flexível formado por pares de arcos cartilaginosos, apresentando epitélio pseudo-estratificado não ciliado no seu revestimento interno, e outros estruturas básicas deste órgão. 0 pulmão se mostrou como um órgão constituído por lobos separados por fissuras com um parênquima usual aos demais mamíferos descritos. No que diz respeito às caraterísticas anatômicas da traqueia e pulmão se mostraram semelhantes à de outros mamíferos, já nos aspectos microscópicos da traqueia apresentou certas peculiaridades diferindo dos demais trabalhos encontrados. No que tange o aspecto do parênquima pulmonar as 
características histológicas não variaram com as que estão descritas.

TERMOS DE INDEXAÇÃO: Traqueia, pulmão, Leopardus pardalis, jaguatirica, árvore bronquial, felinos, parênquima pulmonar, sistema respiratório.

\section{INTRODUÇÃO}

A Jaguatirica (Leopardus pardalis Linnaeus, 1758) é o terceiro maior felino das Américas, de médio porte com peso variando entre 11 e $16 \mathrm{~kg}$ (Murray \& Gardner 1997). No Brasil habita desde florestas úmidas à caatinga, ocorrendo em todos os estados com exceção provável do Rio Grande do Sul (Oliveira \& Cassaro 1999). A jaguatirica, assim como outras seis espécies de felinos brasileiros, está incluída na lista de espécies da fauna brasileira ameaçadas de extinção (IUCN 2013).

O sistema respiratório compreende os pulmões e um sistema de tubos que comunicam o parênquima pulmonar com o meio exterior, possuindo uma porção condutora, formada pelas fossas nasais, nasofaringe, laringe, traqueia, brônquios e bronquíolos, e uma porção respiratória formada pelos bronquíolos respiratórios, ductos alveolares, sacos alveolares e alvéolos pulmonares (Junqueira \& Carneiro 2008).

O estudo comparativo-descritivo da anatomia de animais silvestres está em fase de franco crescimento, no Brasil e em outras partes do mundo, devido ao fato de que o bioma natural de muitos grupos vem sofrendo múltiplas agressões (Leonel et al. 2013), e essas informações contribuem para a área das ciências básicas, as quais podem fornecer informações para a realização de procedimentos clínicos e cirúrgicos, sejam eles de vida livre ou de cativeiro (Heleno et al. 2011).

No que diz respeito à morfologia de animais selvagens, muitas informações ainda são escassas, uma vez que os tratados de anatomia veterinária contemplam, basicamente, animais domésticos (Citrângulo et al. 2001). Diante do es- casso conhecimento sobre estruturas do sistema respiratório de L. pardalis, objetivou-se esclarecer a morfologia da traqueia e pulmão, visando contribuir com conhecimentos que possam auxiliar na conservação da espécie.

\section{MATERIAL E MÉTODOS}

Foram estudados cinco exemplares de jaguatirica (Leopardus pardalis), sendo dois machos e três fêmeas, todos adultos. Dois desses exemplares foram a óbito por atropelamento em rodovias próximas ao município (B.Os 2014.317059(fêmea) e 2015.77025(macho)), dois doados pelo IBAMA após óbito (Registros: 44/09(macho) e 01/15 (fêmea)), e um coletado em óbito no Canteiro de obras UHE-Colíder (MA-0034). Os exemplares foram encaminhados para o Laboratório de Zoologia e Morfologia Animal, UNEMAT-AF pelo IBAMA-AF, fixados em formaldeído a $10 \%$, para estudos morfológicos posteriores. $\mathrm{Na}$ análise macroscópica, foi realizada uma incisão ventral, direção crânio-caudal, na linha Alba, com utilização de instrumental básico para dissecação, com exposição e descrição topográfica dos órgãos. Para microscopia de luz, foram coletados pequenos fragmentos dos órgãos, submetidos à técnica histológica de desidratação em concentrações de álcool (70-100\%), diafanização em xilol e inclusão em parafina histológica e realizados cortes em micrótomo Pat-MRP-12, com espessura média de $5 \mu \mathrm{m}$. A coloração foi realizada a partir das técnicas de Hematoxilina- Eosina (HE). As imagens macroscópicas in situ e ex situ foram realizadas com máquina fotográfica digital FUJI hs20 is, e digitalizadas no programa Microsoft Office Picture Manager 2012 e Microsoft Power Point 2012. As imagens das lâminas histológicas foram fotomicrografadas em microscópico Leica ICC 50 HD. 0 comprimento da traqueia foi mensurado com auxílio de fita métrica e paquímetro $\left(\right.$ Stanley ${ }^{\circledR}$ ) e os valores da média e desvio padrão dispostos em tabelas.

\section{RESULTADOS}

Em seus aspectos macroscópicos, a traqueia se mostrou como um tubo flexível, caudal à laringe, formada por anéis cartilaginosos incompletos, em forma de "C". Estes anéis se apresentaram ligados, na sua porção dorsal, por uma parede musculomembranosa, formando o músculo traqueal,

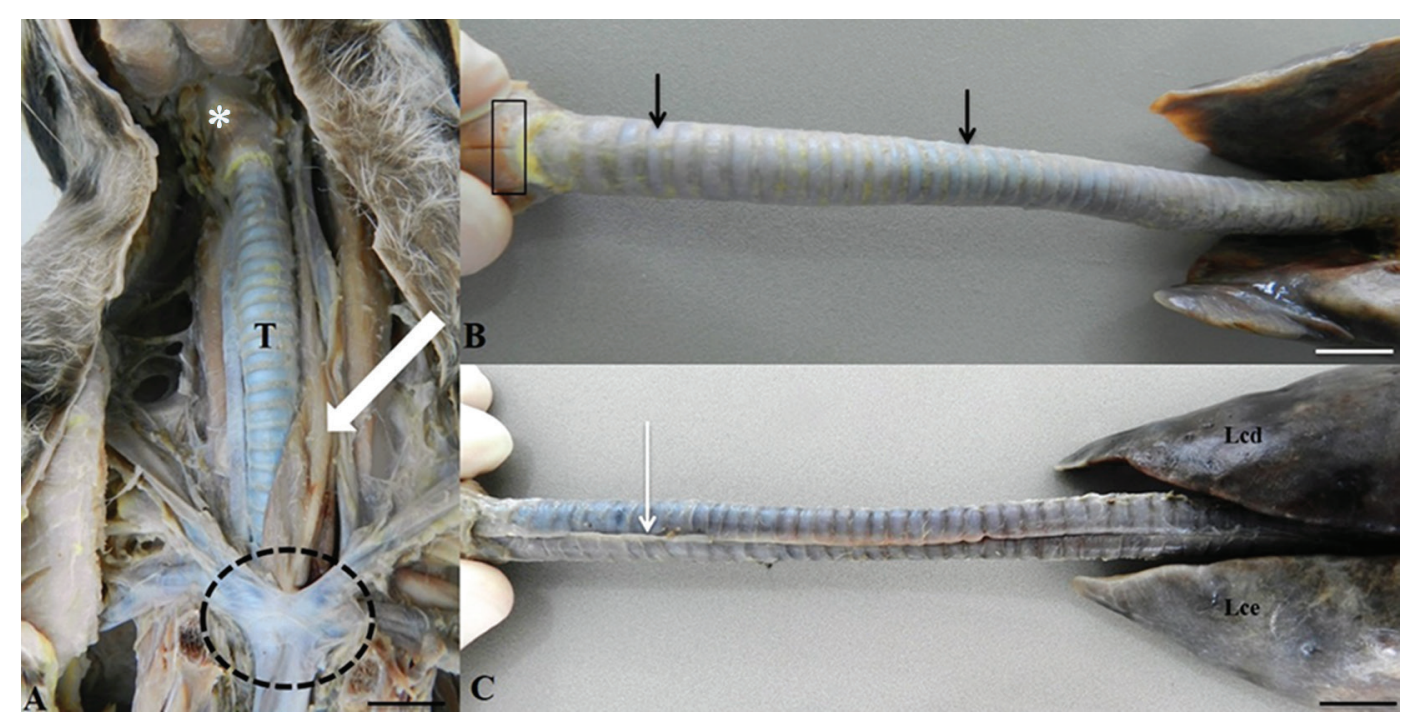

Fig.1. (A) Vista Ventral da traqueia: Laringe (asterisco), traqueia (T), Esôfago (seta grossa), Tronco aórtico (círculo pontilhados). (B ) Borda caudal da cartilagem cricóidea (retângulo), Anéis traqueais cartilaginosos (seta). (C) Vista dorsal da Traqueia: Músculo traqueal (seta branca), Lobo caudal direito (Lcd), Lobo caudal esquerdo (Lce). Barra =: $2 \mathrm{~cm}$. 
unidos aos outros por meio de ligamentos anulares. A traqueia foi dividida, de acordo com sua região no percurso anatômico, em região cervical e torácica. A região cervical localizava-se na porção mediana do pescoço, ventralmente ao esôfago, caudal à laringe, estendendo-se até a altura da C7. A região torácica se iniciava na altura da $\mathrm{T} 1$, onde se relacionava com o início do esôfago torácico, que formava uma curva dorsoventral e apresentava uma leve intumescência nesta região. Esta região da traqueia encontrava-se cranial ao coração e ventral ao arco aórtico (Fig.1). Na sua porção mais caudal se bifurcava formando os brônquios primários. 0 comprimento da traqueia e a quantidade de anéis entre os exemplares variaram, apresentando comprimento médio de $18,9 \mathrm{~cm}$ e quantidade média de anéis de 48,8 (Quadro 1).

A partir da bifurcação caudal da traqueia iniciava-se a formação da árvore brônquica que apresentava dois brônquios principais, o brônquio principal esquerdo e brônquio principal direito, que se ramificam emitindo ramos para os lobos pulmonares, denominados brônquios lobares. Estes se ramificavam em vários brônquios segmentares. 0 brônquio principal direito se ramificava em quatro brônquios segmentares ou secundário, sendo cada um para um lobo pulmonar direito. $\mathrm{O}$ brônquio principal esquerdo bifurcou-

Quadro 1. Comprimento traqueal e número total de anéis traqueais de Leopardus pardalis

\begin{tabular}{lcc}
\hline Espécime & Comprimento traqueal $(\mathrm{cm})$ & Anéis traqueais \\
\hline 1 Fêmea & 17 & 49 \\
2 Macho & 20 & 47 \\
3 Fêmea & 20 & 50 \\
4 Fêmea & 18 & 49 \\
5 Macho & 19,5 & 49 \\
Média & 18,9 & 48,8 \\
Desvio Padrão & 1,34 & 1,09
\end{tabular}

-se em dois ramos segmentares, um para o lobo cranial e um para o lobo caudal (Fig.2).

Os pulmões se apresentaram como órgãos de consistência esponjosa, de coloração avermelhada, com sua base relacionada à superfície torácica do diafragma e seu ápice com a região apical do tórax, formado por lobos separados por fissuras interlobares bem evidentes. Em L. pardalis o pulmão direito estava dividido em quatro lobos, o lobo cranial, médio, caudal e acessório direito, enquanto o pulmão esquerdo se constituía por dois lobos, lobo cranial e caudal esquerdo. No lobo cranial direito havia uma incisura intralobar, dividindo-o em porção cranial e caudal do lobo pulmonar direito (Fig.3).

As análises microscópicas mostraram que a traqueia se constituía por dois arcos cartilaginosos cujas extremidades dorsais se sobrepunham, formando um arcabouço que permite uma eficaz movimentação ao órgão, ligados por uma camada de tecido conjuntivo frouxo modelado e musculo liso. As extremidades ventrais eram livres, unidas entre si por uma membrana constituída por epitélio pseudo-estratificado não ciliado, tecido conjuntivo frouxo não modelado, uma camada de conjuntos de ácinos serosos seguidos de uma fina camada de tecido conjuntivo frouxo modelado e uma espessa camada de músculo liso, região denominada de músculo traqueal. Revestindo sua superfície externa, uma membrana constituída por bi-epitélio pavimentoso. Esta membrana revestia toda a superfície interna da traqueia, mantendo suas características morfológicas até alcançarem a superfície interna da cartilagem. A cartilagem se mostrou do tipo hialina, com presença de condrócitos e condroblastos imersos na matriz cartilaginosa formando grupos isógenos. Sobre a superfície externa da cartilagem observou-se o pericôndrio seguido de feixes de músculos lisos. Ao logo da extensão de cada arco havia regiões de leves constrições com presença de células formadoras de

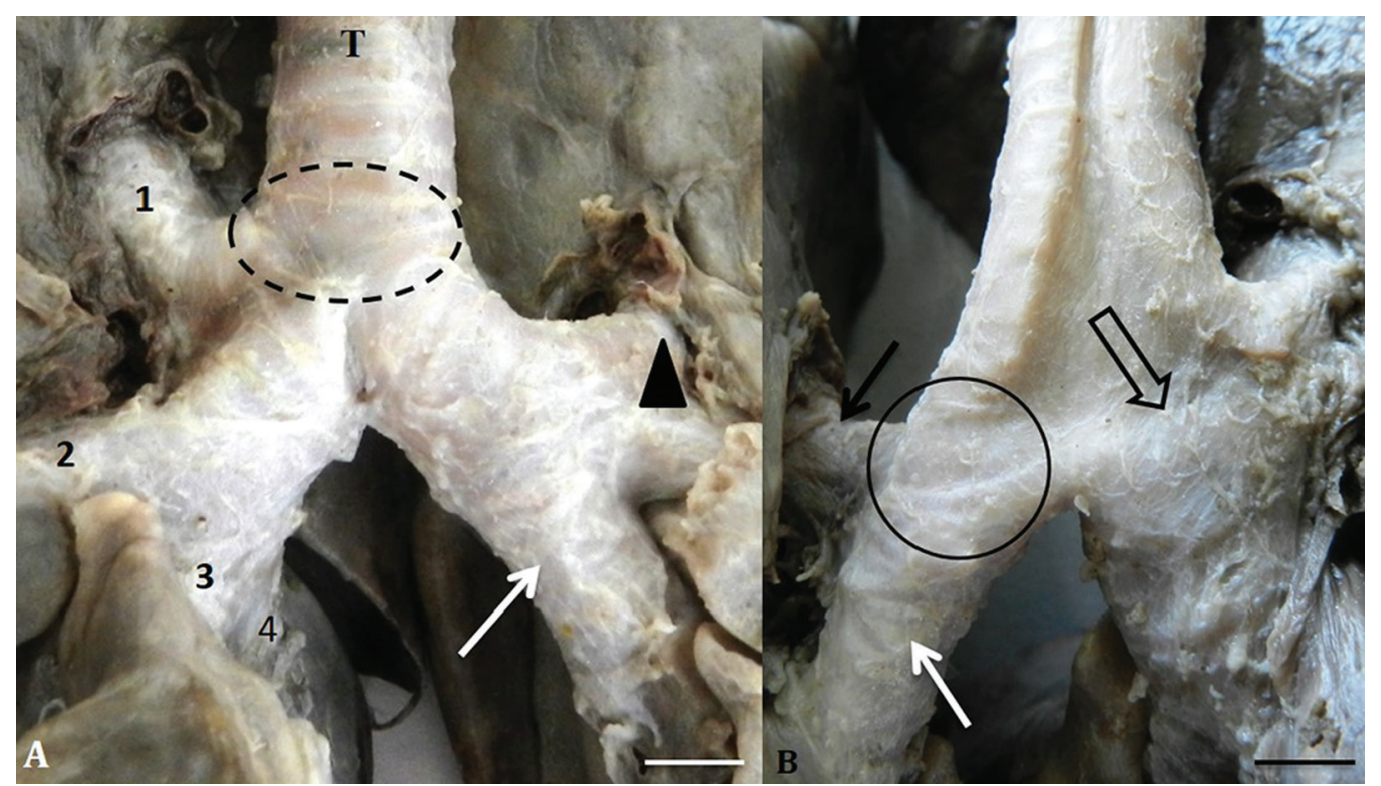

Fig.2. (A) Vista ventral: Traqueia (T), Carina traqueal (círculo pontilhado), Brônquio lobar cranial direito (1), Brônquio lobar medial direito (2), Brônquio lobar acessório direito (3), Brônquio lobar caudal direito (4), Brônquio lobar cranial esquerdo (cabeça de seta), Brônquio lobar caudal esquerdo (seta branca). (B) Vista dorsal: Brônquio principal direito (seta vazada), Brônquio principal esquerdo (círculo), Brônquio lobar cranial esquerdo (seta preta), Brônquio lobar caudal esquerdo (seta branca). Barra = $2 \mathrm{~cm}$. 


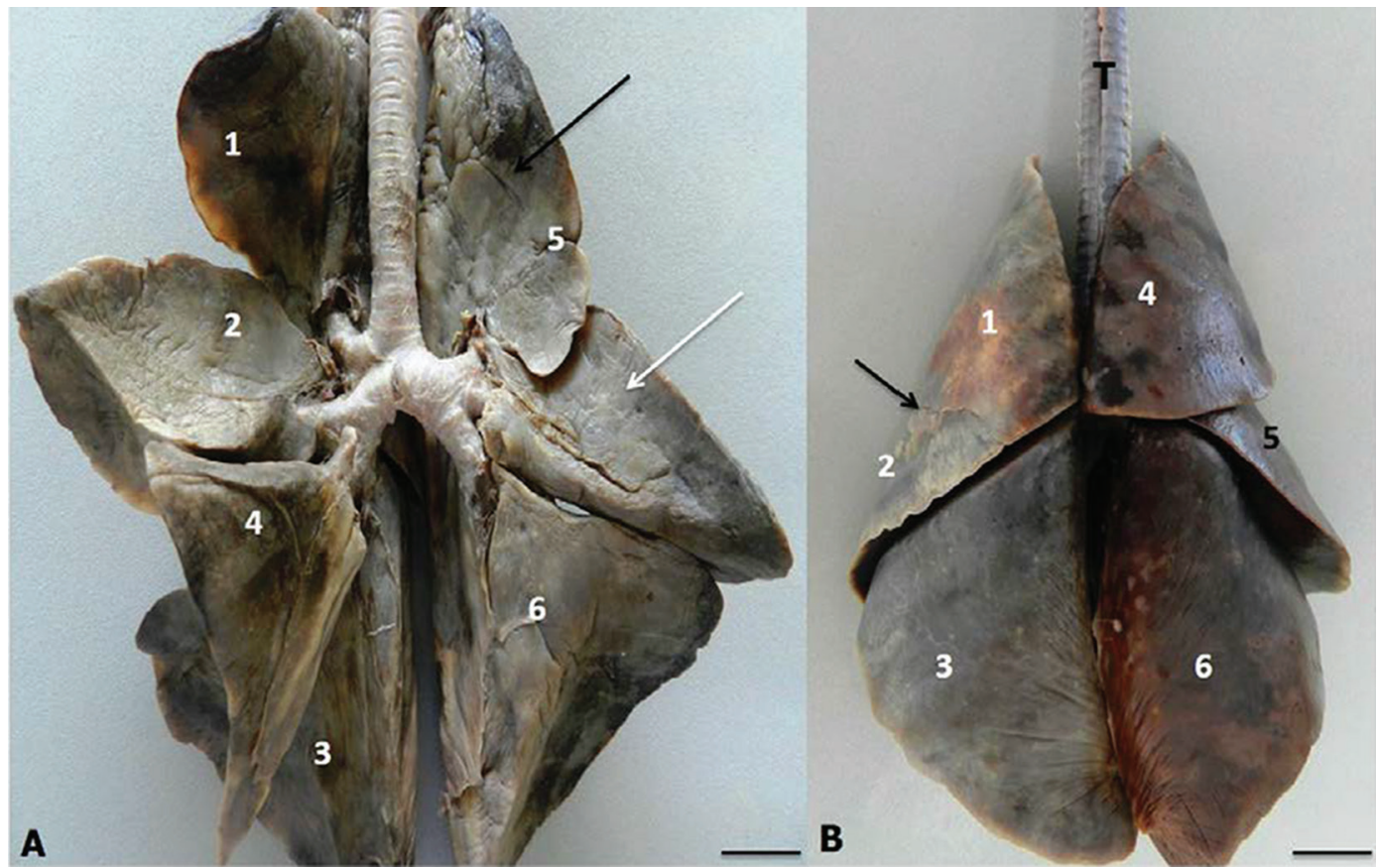

Fig.3. Vista ventral Pulmão Leopardus pardalis: (A) Lobo pulmonar cranial direito (1), Lobo pulmonar medial direito (2), Lobo pulmonar caudal direito (3), Lobo pulmonar acessório (4), Lobo pulmonar cranial esquerdo (5) e suas respectivas regiões cranial (seta preta) e caudal (seta branca), Lobo pulmonar caudal esquerdo (6). Vista dorsal: (B) Traqueia (T), Fissura intralobar (seta) dividindo o Lobo cranial esquerdo em região cranial (1) e região caudal (2), Lobo caudal esquerdo (3), Lobo pulmonar cranial direito (4), Lobo pulmonar medial direito (5), Lobo pulmonar caudal direito (6). Barra $=2 \mathrm{~cm}$.

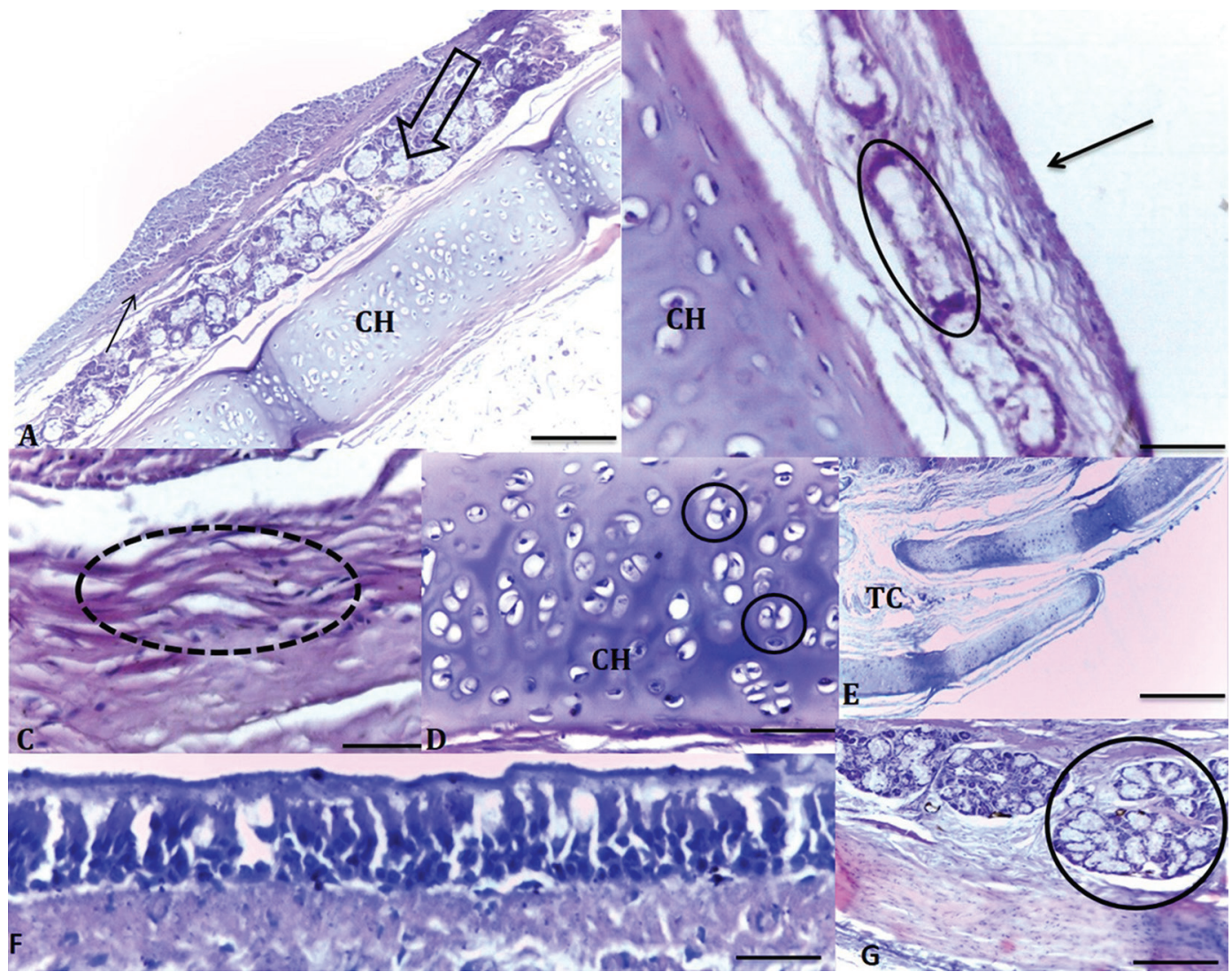

Fig.4. (A) Tecido conjuntivo (seta fina), Ácinos serosos (seta vazada), Cartilagem hialina (CH), Constrições na cartilagem (cabeça de seta). (B) Epitélio da traqueia (seta fina), Ácino seroso (círculo), Cartilagem hialina (CH). (C) Musculo liso (círculo pontilhado). (D) Cartilagem hialina $(\mathrm{CH})$, Condrócitos formando grupos isógenos (círculo). (E) Observar o epitélio pseudo-estratificado, Tecido conjuntivo (TC). (F) Epitélio pseudo-estratificado. (G) Ácinos serosos (círculo). HE, Barra = $25 \mu \mathrm{m}$. 


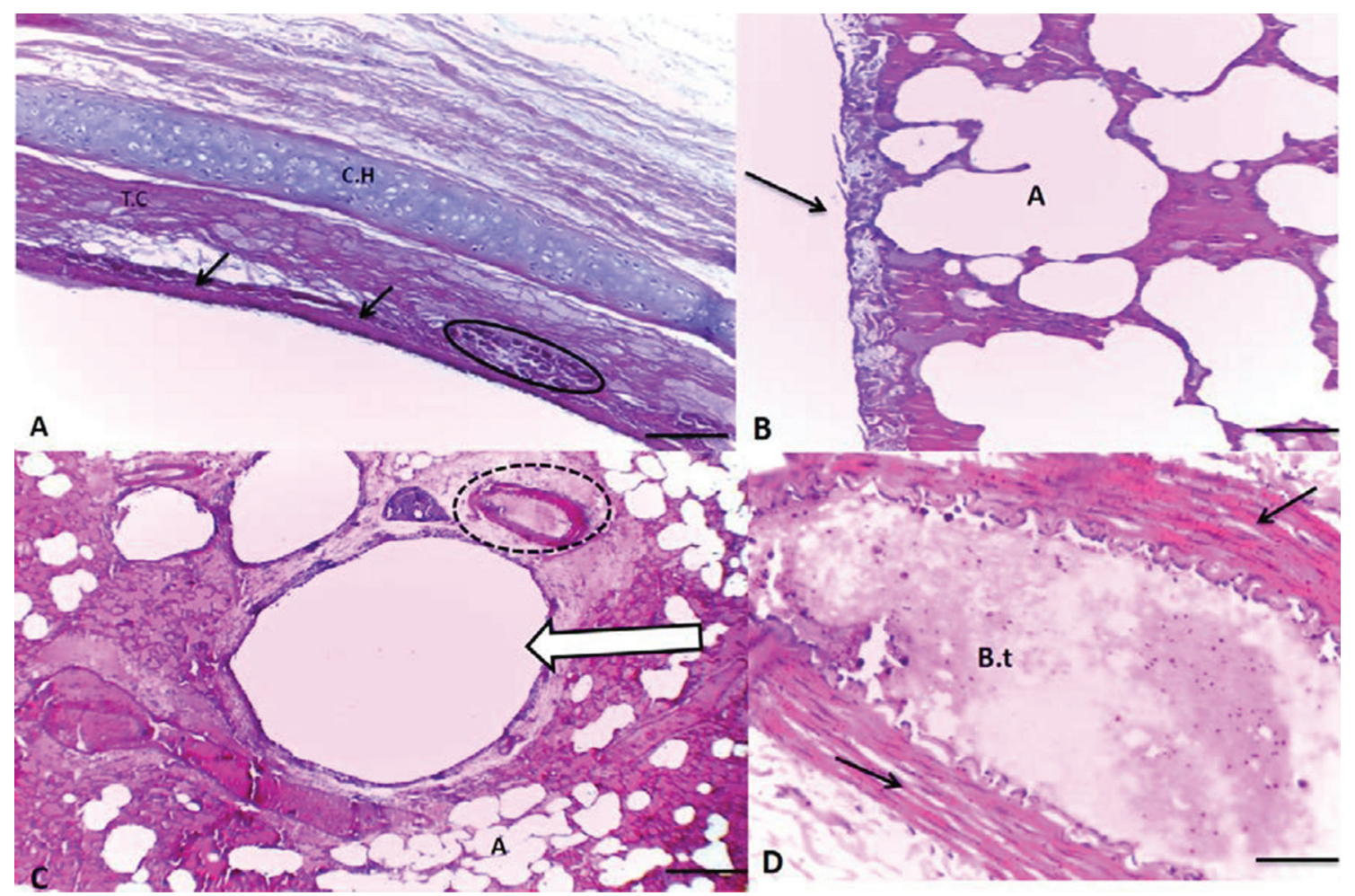

Fig.5. (A) Brônquio principal: Músculo liso (setas), Ácinos (círculo), Tecido conjuntivo (TC), Cartilagem hialina (CH). (B) Parênquima pulmonar: Tecido conjuntivo (seta), Sacos alveolares (A). (C) Alvéolos (A), Brônquio segmentar (seta branca), Bronquíolo terminal (círculo pontilhado). (D) Músculo liso (seta fina), Bronquíolo terminal (BT). HE, Barra $=25 \mu \mathrm{m}$.

cartilagem, os condrócitos, indicando uma área de produção celular (Fig.4). Não foi encontrada diferença nos aspectos microscópicos entre os arcos das regiões cervical e torácica da traqueia. A mesma constituição histológica foi observada nos brônquios principais.

Histologicamente os lobos pulmonares se mostraram revestidos por uma fina camada de tecido conjuntivo, com o parênquima pulmonar constituído por brônquios que se afunilavam formando os bronquíolos. Seu revestimento interno era formado por um epitélio cúbico simples, revestidos por um anel de músculo liso. Os sacos alveolares se encontravam aglomerados apresentando forma de rosetas, dispersos em uma fina camada de tecido conjuntivo frouxo, vasos sanguíneos e músculo liso (Fig.5).

\section{DISCUSSÃO}

Nos seus aspectos macroscópicos a traqueia de Leopardus pardalis é similar a das espécies de animais silvestres como Sapajus apela (Leonel et al. 2013), Nasua nasua (Oliveira et al. 2012), Saimiri sciureus (Pinheiro et al. 2012) e carnívoros domésticos (Dyce et al. 2010), diferindo de Bradypus variegatus (Faro et al. 2015) que apresentava sinuosidades em sua porção médio caudal. A quantidade de anéis e comprimento traqueal variou entre os espécimes estudados, assim como em Didelphis sp. (Bertassoli et al. 2013) e B. variegatus (Faro et al. 2015). Segundo Getty (1986), a traqueia do cão tem uma variação de 42 a 46 anéis traqueais, enquanto a dos gatos possue entre 38 a 43. Dyce et al. (2010), relatam que os números de anéis traqueais são diversos, nos mamíferos por exemplo podem variar de 29 a 60.
Em Leopardus pardalis o pulmão era dividido externamente por fissuras bastante evidentes correspondendo às divisões dos lobos, tanto à direita quanto à esquerda, assim como em outras espécies de vertebrados descritos na literatura como carnívoros domésticos (Dyce et al. 2010), Puma yagouaroundi (Santos et al. 2011), Conepatus semistriatus (Rosa et al. 2012) Procyon cancrivorus (Sestari et al. 2011, Paranaíba et al. 2016). As fissuras interlobares permitem o deslizamento das partes uma sobre a outra, o que torna mais fácil a adaptação dos pulmões nas mudanças da forma torácica quando os animais se exercitam (Dyce et al. 2010).

A lobação pulmonar varia entre os vertebrados, os espécimes estudados apresentaram a mesma lobação descrita para Dasyprocta sp. (Penno et al. 2005), Sphiggurus villosus (Guimarães et al. 2012), Hydrochaeris hydrochaeris (Citrângulo et al. 2001) e P. yagouaroundi (Santos et al. 2011) em que o pulmão direito apresentou lobo cranial, médio, caudal e acessório e o pulmão esquerdo um lobo cranial, dividido em segmentos cranial e caudal pela incisura intralobar, e lobo caudal. Nas espécies $P$. cancrivorus (Sestari et al. 2011, Paranaíba et al. 2016), C. semistriatus (Rosa et al. 2012) e Mazama gouazoubira (Martins et al. 2014), foi relatado que o pulmão esquerdo possui dois lobos, cranial e caudal, não havendo a presença da incissura intralobar. Nos marsupiais Monodelphis domestica e Didelphis virginiana foi descrito que o pulmão esquerdo não era separado por fissuras profundas, apresentado um único lobo que foi dividido em região cranial e caudal (Cope et al. 2001). Em Cuniculus paca o pulmão esquerdo possuía três lobos bem 
delimitados, sendo um lobo cranial bilobado em partes cranial e caudal, um lobo caudal e um pequeno lobo acessório (Rehder et al. 2008).

Segundo Santos et al. (2013) a lobação pulmonar parece seguir um padrão de distribuição entre mamíferos da ordem Carnívora, fato este não evidenciado em nosso estudo com L. pardalis, espécie carnívora que apresentou uma lobação do pulmão esquerdo diferente, pois apresentava a fissura intralobolar no lobo cranial esquerdo, o que defere de outros carnívoros descritos como gatos (Oliveira et al. 2001), cães (Dyce et al. 2010), N. nasua (Oliveira et al. 2012) P. cancrivorus (Sestari et al. 2011, Santos et al. 2013, Paranaíba et al. 2016).

A distribuição da árvore brônquica direita é a mesma descrita para $D$. virginiana (Cope et al. 2001) e P. cancrivorus (Paranaíba et al. 2016) que apresentaram brônquios lobares cranial, medial, acessório e caudal, diferindo da relatada para o S. villosus (Guimarães et al. 2012), onde o brônquio principal direito se trifurcava em um brônquio lobar para o lobo cranial, um para o médio e um para os lobos caudal e acessório. A distribuição esquerda é a mesma descrita para alguns roedores, como Dasyprocta sp., S. villosus e H. hydrochaeris (Penno et al. 2005, Citrângulo et al. 2001, Guimarães et al. 2012), e de alguns carnívoros como P. yagouaroundi, C. semistriatus (Santos et al. 2011, Rosa et al. 2012). Em L. pardalis não foi observado o brônquio traqueal presente no cervídeo M. gouazoubira (Martins et al. 2014), ovinos raça ideal (Borges et al. 2002), e fetos de bovinos azebuados (Trindade et al. 2003).

Nos aspectos microscópicos a traqueia diferiu em relação ao seu epitélio, que se mostrou não ciliado, as demais estruturas constituintes dos órgãos eram similares a Bradypus variegatus (Faro et al. 2015), N. nasua (Oliveira et al. 2012), Cricetomys gambianus (Ibe et al. 2011) e Vulpes vulpes (Moussa \& Hassan 2015). Em relação às constrições nos arcos de cartilagens não foi encontrada na literatura nenhuma informação referente a esta característica. Assim como a traqueia, os brônquios não apresentaram um epitélio ciliado, diferindo dos trabalhos encontrados na literatura como P. cancrivorus (Paranaíba et al. 2016), Cricetomys gambianus (Ibe et al. 2011) e N. nasua (Oliveira et al. 2012).

Os anéis traqueais em Didelphis sp. (Bertassoli et al. 2013) apresentaram forma de " $C$ ", com músculo liso completando a circunferência, os quais estavam dispostos em faixas transversais que uniam as extremidades dorsais das cartilagens. A presença de músculo liso, ligando as extremidades dos arcos, também foi observada em L. pardalis.

Em relação ao parênquima pulmonar as características histológicas não variaram com as que estão descritas na literatura para animais domésticos (Samuelson 2007), Cricetomys gambianus (Ibe et al. 2011) e P. cancrivorus (Paranaíba et al. 2016).

\section{CONCLUSÕES}

A estrutura anatômica da traqueia e a lobação pulmonar de Leopardus pardalis não apresentaram grandes divergências em relação as suas características, sendo similar com animais domésticos e silvestres descritos na literatura.
Os aspectos histológicos da traqueia diferiram quanto ao epitélio, que se mostrou não ciliado; isto pode ter sido devido à demora na fixação dos exemplares, que ocorreu em média 14 horas após as morte.

Os demais constituintes microscópicos da traqueia, brônquios e dos lobos pulmonares não apresentaram diferenças com a literatura consultada.

Agradecimentos.- 0 primeiro autor agradece à Coordenação de Aperfeiçoamento de Pessoal de Nível Superior (CAPES) pela concessão da bolsa de mestrado.

\section{REFERÊNCIAS}

Bertassoli B.M., Santos A.C., Oliveira F.D., Oliveira D.M., Assis-Neto A.C \& Carvalho A.F. 2013. Morfologia da laringe e traqueia de gambás (Didelphis sp.). Ciênc. Anim. Bras, 14 (2):222-229.

Borges E.M., Oliveira F.S., Machado M.R.F., Ribeiro A.A.C.M. \& Silva-Sobrinho A.G. 2002. Segmentação anatomocirúrgica arterial dos pulmões de ovinos da raça ideal (Ovis aires Linaeus, 1758). Braz. J. Vet. Res. Anim. Sci., 39(6):288-295.

Citrângulo M., Ribeiro A.A.C.M., Moraes P.T.B. \& Machado M.R.F. 2001. Lobation and arterial vascularization of the lung of capybara (Hydrochaeris hydrochaeris). Arq. Ciênc. Vet. Zool., 4 (2):119-127.

Cope L.A., Henry R.W. \& Reed Jr R.B. 2001. Macroscopic anatomy of the lower respiratory tract of the gray short-tailed opossum (Monodelphis domestica). Braz. J. Morphol. Sci. 18(2):111-117.

Dyce K.M., Sack W.O. \& Wensing C.J. G 2010. Tratado de Anatomia Veterinária. 5a ed. Elsevier, Rio de Janeiro. 813p.

Faro T.A.S., Lima A.R., Messias A.C., Cabral R., Giese E.G., Matos E.R. \& Branco E. 2015. Morfologia e morfometria da traqueia da preguiça (Bradypus variegatus): conhecimentos para procedimentos de emergência. Pesq. Vet. Bras. 35(2):193-198.

Getty R. 1986. Sisson and Grossman's Anatomy of the Domestic Animals. 5th ed. W.B. Saunders, Philadelphia. 1211p.

Guimarães G.C., Lopes G.C., Rosa M.C.B., Sestari C.E.O. \& Oliveira F.S. de. 2012. Lobação pulmonar e distribuição brônquica do ouriço-cacheiro (Sphiggurus villosus). Acta Scient. Vet. 40(2):1037-1041.

Heleno A.R., Santos L.M., Miglino M.A., Peres J.A. \& Guerra R.R. 2011. Biometria, histologia e morfometria do sistema digestório do cachorro-do-mato (Cerdocyon thous) de vida livre. Biotemas 24(4):111-119.

Ibe C.S., Onyeanusi B.I., Salami O. \& Nzalak J.O. 2011. Microscopic anatomy of the lower respiratory system of the African giant pouched rat (Cricetomys gambianus Waterhouse, 1840). Int. J. Morphol. 29(1):27-33.

IUCN 2013. IUCN Red List of Threatened Species. Version 2013.2. Disponível em <http://www.iucnredlist.org> Acesso em 6 abril de 2016.

Junqueira L.C. \& Carneiro J. 2008. Histologia Básica. 11ํㅗ ed., Guanabara Koogan, Rio de Janeiro. 524p.

Leonel L.C.P.C., Lima T.C., Felipe R.L., Silva E.M., Silva G.A.O., Silva D.C.O., Carvalho-Barros R.A. \& Silva Z. 2013. Anatomia descritiva da traqueia do macaco-prego (Sapajus apella). Biotemas 26(4):179-183.

Martins T.M.M., Pereira K.F., Lima F.C., Fontana C.A.P., Santos A.L.Q. \& Malysz T. 2014. Distribuição dos brônquios lobares dos pulmões do veado-catingueiro Mazama gouazoubira. Pesq. Vet. Bras. 34(5):473-476.

Murray J.L. \& Gardner G.L. 1997. Leopardus pardalis. Mammalian Species 548:1-10.

Moussa E.A. \& Hassan A.S. 2015. Histology and scanning electron microscopy of the lower respiratory tract in the adult red fox (Vulpes vulpes). Int. J. Morphol, 33(1):267-274.

Oliveira F.S., Borges E.M., Machado M.R.F., Canola J.C. \& Ribeiro A.A.C.M. 2001. Segmentação anátomo-cirúrgica arterial dos pulmões de gato (Felis catus domesticus L., 1758). Braz. J. Vet. Res. Anim. Sci, 38(6):253257.

Oliveira T.G. \& Cassaro K. 1999. Guia de Identificação dos Felinos Brasileiros. $2^{\underline{a}}$ ed. Sociedade de Zoológicos do Brasil. Atheneu, São Paulo. 560p. 
Oliveira V. C., Souza A.F., Santos A.C., Bertassoli B.M., Rosa R.A., Carvalho A.F., Martins J.F. \& Mançanares C.A.F. 2012. Estudo morfológico do sistema respiratório de quati (Nasua nasua). Biotemas 25(1):81-92.

Paranaíba J.F.F.S., Lima F.C., Pereira D.K.S., Araújo E.G., \& Pereira K.F. 2016. Morfo-histologia dos pulmões e árvore bronquial de Procyon cancrivorus (Carnivora: Procyonidae). Arq. Bras. Med. Vet. Zootec. 68(2):353-360.

Penno A.K., Carvalho M.A.M., Assis Neto A.C., Azevedo L.M. \& Mello G.W.S. 2005. Lobação, ramificação brônquica e distribuição arterial no pulmão da cutia (Dasyprocta sp., Mammalia, Rodentia). Braz. J. Vet. Res. Anim. Sci. 42(5):327-332.

Pinheiro L.L., Lima A.R., Muniz J.A.P.C., Imbeloni A., Fioreto E.T., Fontes R.F., Cabral R. \& Branco E. 2012. Anatomy and morphometric aspects of the trachea of Saimiri sciureus Linnaeus, 1758: knowledge for emergency procedures. An. Acad. Bras. Ciênc. 84(4):973-977.

Rehder A.M.A., Cortellini L.M., Oliveira F.S. \& Machado M.R.F. 2008. Lobação, árvore brônquica e vascularização arterial do pulmão da paca (Cuniculus paca Linnaeus, 1766). Ciênc. Anim. Bras. 9(2):442-448.

Rosa M.C.B., Lopes G.C., Sestari C.E.O., Guimarães G.C. \& Oliveira F.S. 2012. Lo- bação pulmonar e distribuição brônquica da jaritataca (Conepatus semistriatus Boddaert, 1785): comunicação curta. Vet. Zootec. 19(3):332- 336.

Santos A.C., Oliveira V.C., Bertassoli B.M., Lobo L.M., Viana D.C., Vasconcelos B.G., Assis Neto A.C., Carvalho A.F. \& Mançanares C.A.F. 2013. Morfologia macroscópica dos pulmões e distribuição bronquiopulmonar em mão-pelada (Procyon cancrivorus). Biotemas 26(4):195-202.

Santos A.L.Q., Moraes F.M., Carvalho S.F.M., Menezes L.T., Kaminishi A.P.S., Leonardo T.G. \& Nascimento L.R. 2011. Lobos pulmonares e formação dos brônquios do gato mourisco (Puma yagouaroundi Severtzow, 1848) (Felidae). PUBVET 5(13), Art.1083.

Samuelson D.A. 2007. Tratado de Histologia Veterinária. Elsevier, Rio de Janeiro, $527 \mathrm{p}$.

Sestari C.E.O., Corrêa A.F., Martins L.L., Guimarães G.C. \& Oliveira F.S. 2011. Lobação pulmonar e distribuição brônquica em Mão-Pelada (Procyon cancrivorus Cuvier, 1798): relato de dois casos. Vet. Zootec. 18(3):374378.

Trindade L.B., Andrade E.A.A. \& Melo A.P.F. 2003. Segmentação do pulmão de fetos de bovinos azebuados. Braz. J. Vet. Res. Anim. Sci. 40(2):95-99. 